\section{Mechanical Control of Spin States and Conductance Achieved in Cobalt Complex}

Progress in the ability to fabricate controllable single-molecule electrical devices has enabled these devices to be used as scientific tools to perform detailed measurements of electron correlations on nanometer-length scales. Recently, D.C. Ralph of Cornell University; T.A. Costi of Forschungszentrum Jülich, Germany; P.S. Cornaglia of Consejo Nacional de Investigaciones Científicas y Técnicas (CONICET), Argentina; and their colleagues have studied the spin states in single molecules attached to gold electrodes and the Kondo effect by which the molecular spin can be screened by electrons, as a function of stretching the molecule. Electronic states depend on symmetry; for example, a complex composed of a transition metal and ligands breaks spherical symmetry and splits the metal's originally degenerate d orbitals. For spin $S>1$, distortion of the ligand geometry causes additional splitting of the $(2 S+1)$-degenerate spin states, resulting in magnetic anisotropy.

The researchers modified the symmetry of a spin $S=1$ Co complex by controllably stretching it, and simultaneously measuring the current flowing through it. They were able to use mechanical control of the Co complex to demonstrate the underscreened Kondo effect, where conduction electrons only partially screen the molecular spin, from $S=1$ to $1 / 2$.

As reported in the June 11th issue of Science (DOI: 10.1126/science.1186874; p. 1370), the researchers used mechanically controllable break-junction devices to stretch individual $\mathrm{Co}$ (tpy-SH) ${ }_{2} \mathrm{com}$ plexes (where tpy-SH is $4^{\prime}$-mercapto$2,2^{\prime}: 6^{\prime}, 2^{\prime \prime}$-terpyridine). The researchers measured differential conductance $(d I / d V)$ as a function of increasing electrode separation at a temperature of $1.6 \mathrm{~K}$.

Initially, the spectra displayed the typical signature of Kondo-assisted tunneling through the molecule-a single peak centered at $V=0$. However, as the molecule was stretched beyond a certain devicedependent point, the peak split into two, in contrast to the spin $S=1 / 2$ Kondo effect in $\mathrm{C}_{60}$ molecules, where no peak splitting was observed.

The researchers showed that the peak splitting observed for the Co complex is due to a higher spin $S=1$ Kondo effect and degeneracy breaking within the triplet ground state caused by molecular distortion. The researchers said that their work makes possible "dramatically more detailed and precise comparisons with theory," and that it, "further demonstrates that mechanical control can be a realistic strategy for manipulating molecular spin states to supplement or replace the use of magnetic fields in proposed applications such as quantum manipulation or information storage."

STEVEN TROHALAKI

\section{Defect-as-Qubit Paradigm Developed in New Materials Systems}

Quantum computing is one of the most tantalizing promises of modern physics. Its actualization hinges on finding suitable materials systems for storing and manipulating bits of quantum information, known as qubits. An approach suggested in recent years is to use bound nitrogen-vacancy defect states in diamond, which enable manipulation and high-fidelity measurement of qubits at room temperature. Implementing these qubits in a quantum computer presents a formidable challenge, given the difficulty of growing and fabricating diamond, but researchers at the University of California at Santa Barbara have brought the science a step closer to technology by expanding the defect approach to other, more accessible host materials.
The nitrogen-vacancy defect in diamond $\left(\mathrm{N}_{\mathrm{C}} \mathrm{V}_{\mathrm{C}}^{-1}\right)$ is a bound state of several electrons that can undergo certain spin transitions, allowing it to be pumped and probed optically in order to "read" and "write" the qubit. To identify similar systems in other materials, J.R. Weber, W.F. Koehl, J.B. Varley, A. Janotti, B.B. Buckley, C.G. Van de Walle, and D.D. Awschalom developed a list of criteria for both the defect centers and the host materials, simultaneously developing the paradigm of defects as qubits and providing a roadmap for exporting the diamond NV approach to other systems. As published in the May 11th issue of the Proceedings of the National Academy of Sciences (DOI: 10.1073/ pnas.1003052107; p. 8513), their criteria address issues of initializing and reading the qubits, as well as ensuring long lifetimes and thermal stability.
The team then applied the criteria to defect systems in diamond and the $4 \mathrm{H}$ polytype of $\mathrm{SiC}$. The researchers used density functional theory (DFT) to calculate defect stabilities, charge, and spin states, employing hybrid functionals to accurately determine bandgaps and constrained DFT to calculate defect excitation energies. An analysis of the results reveals that the required features, primarily the existence of a ground-state spin triplet, may be supported by certain $\mathrm{N}_{C} \mathrm{~V}_{\mathrm{Si}}$ systems in $\mathrm{SiC}$, as well as by isolated vacancies such as $\mathrm{V}_{\mathrm{Si}}{ }^{-2}$. Taking these results as a starting point, the researchers extend their discussion to analogous defect centers in other semiconductors. Their work paves the way for a broad expansion of the defect-as-qubit paradigm and brings quantum computing one step closer to reality.

Alison HatT

\section{High Quality Photoactive Cubic- Phase Copper Selenide Nanodiscs Show Promise for Photovoltaics}

Copper selenide is a promising candidate for active components in solar cells because it contains cheap and abundant elements and has a bandgap in an optimal range to absorb energy from the solar spectrum. Colloidal nanomaterials may enable inexpensive, large area fabrication through solution processing. However, current copper selenide nanoparticle syntheses often use phosphinebased precursors which, in addition to being toxic and oxygen sensitive, tend to result in polydisperse size and shape of nanomaterial reaction products. Recently, J. Choi, N. Kang, H.Y. Yang, and S.U. Son from Sungkyunkwan University, Suwon, Korea, and H.J. Kim from the Korea Basic Science Institute, Daejeon, Korea, report a solution-based approach to obtaining high-quality cubic phase copper selenide nanodiscs of uniform size and shape as published in the June 22nd issue of Chemistry of Materials (DOI: 10.1021/ cm100902f; p. 3586).

The researchers generated a new, air stable selenium precursor, 1,3 dimethylimidazoline-2-selenone using $\mathrm{N}$-heterocyclic carbene (NHC) chemistry. Nanoparticle synthesis using this precursor was optimized for solvent content, reactant concentration, and temperature. The nanodiscs obtained in their optimal process were characterized by transmission electron microscopy, energydispersive spectroscopy, $x$-ray powder diffraction, and x-ray photoelectron spectroscopy. The discs are cubic phase $\mathrm{Cu}_{2-x}$ Se with diameters of $17( \pm 1) \mathrm{nm}$ and thicknesses of $2.6( \pm 1) \mathrm{nm}$, making these 